\title{
Note on the Interactive Elements of the Project
}

Some of the experimental interactive artefacts which have been produced as part of the research for this book can be downloaded from shotscarecrow.itch.io. They may, in the months and years since parts of the book were written, have reached slightly more advanced states than as described. 